\title{
Risk factors for acute kidney injury after pediatric cardiac surgery: a meta-analysis
}

\author{
Jef Van den Eynde ${ }^{1,2}$ (D) Boris Delpire ${ }^{2} \cdot$ Xander Jacquemyn $^{2} \cdot$ Ismat Pardi $^{2} \cdot$ Hajar Rotbi $^{3,4} \cdot$ Marc Gewillig $^{2,5}$. \\ Shelby Kutty ${ }^{1}$. Djalila Mekahli ${ }^{6,7}$
}

Received: 30 June 2021 / Revised: 16 September 2021 / Accepted: 16 September 2021

(c) The Author(s), under exclusive licence to International Pediatric Nephrology Association 2021

\begin{abstract}
Background Cardiac surgery-associated acute kidney injury (AKI) is associated with increased morbidity and mortality in both adults and children.

Objectives This study aimed to identify clinical risk factors for AKI following cardiac surgery in the pediatric population. Data sources PubMed/MEDLINE, Embase, Scopus, and reference lists of relevant articles were searched for studies published by August 2020.

Study eligibility criteria Studies were included if (1) the population consisted of pediatric patients ( $<18$ years old), (2) patients underwent cardiac surgery, (3) risk factors were compared between patients who developed AKI and those who did not, and (4) studies were prospective or retrospective observational studies or randomized controlled trials.

Participants and interventions Children undergoing pediatric cardiac surgery.

Study appraisal and synthesis methods Random-effects meta-analysis was performed, comparing potential risk factors between pediatric patients who developed CS-AKI and those who did not.

Results Sixty-one publications including a total of 19,680 participants (AKI: 7257 participants; no AKI: 12,423 participants) were included from studies published between 2008 and 2020. The pooled estimated incidence of AKI was $34.3 \%$ (95\% confidence interval $30.0-38.8 \%, I^{2}=96.8 \%$ ). Binary risk factors that were significantly and consistently associated with AKI were the presence of pulmonary hypertension, cyanotic heart disease, univentricular heart, risk adjustment for congenital heart surgery 1 (RACHS-1) score $\geq 3$, vasopressor use, cardiopulmonary bypass use, reoperation, and sepsis. Significant continuous risk factors included younger age, lower body weight, lower preoperative creatinine, higher preoperative estimated glomerular filtration rate (eGFR), higher RACHS-1 score, longer surgery time, longer cardiopulmonary bypass time, longer aortic cross-clamp time, and higher red blood cell transfusion volume.

Limitations Results are limited by heterogeneity and potential residual confounding.

Conclusions and implications of key findings Our meta-analysis identified clinical risk factors that are associated with AKI in children undergoing cardiac surgery. This might help clinicians anticipate and manage more carefully this population and implement standardized preventive strategies.
\end{abstract}

Systematic review registration number CRD42021262699.

Keywords Acute kidney injury $\cdot$ Cardiac surgery $\cdot$ Meta-analysis $\cdot$ Pediatric $\cdot$ Risk factors

\begin{tabular}{ll} 
Abbreviations \\
AKI & Acute kidney injury \\
AKIN & Acute kidney injury network \\
$\mathrm{CI}$ & Confidence interval \\
$\mathrm{CPB}$ & Cardiopulmonary bypass \\
$\mathrm{eCrCl}$ & Estimated creatinine clearance \\
& \\
\hline & Jef Van den Eynde \\
jef.vandeneynde@ student.kuleuven.be
\end{tabular}

\section{Abbreviations}

AKI

AKIN

CI

$\mathrm{CPB}$

Published online: 30 September 2021

\begin{tabular}{|c|c|}
\hline eGFR & Estimated glomerular filtration rate \\
\hline KDIGO & Kidney disease improving global outcomes \\
\hline MD & Mean difference \\
\hline MOOSE & $\begin{array}{l}\text { Meta-analysis of observational studies in } \\
\text { epidemiology }\end{array}$ \\
\hline OR & Odds ratio \\
\hline PRISMA & $\begin{array}{l}\text { Preferred reporting items for systematic } \\
\text { reviews meta-analyses }\end{array}$ \\
\hline PROSPERO & $\begin{array}{l}\text { International prospective register of sys- } \\
\text { tematic reviews }\end{array}$ \\
\hline
\end{tabular}




$\begin{array}{ll}\text { RACHS-1 } & \begin{array}{l}\text { Risk adjustment for congenital heart sur- } \\ \text { gery } 1\end{array} \\ \text { RIFLE } & \begin{array}{l}\text { Risk for renal dysfunction, Injury to the } \\ \text { kidney, failure of kidney function, loss of } \\ \text { kidney function and end-stage renal disease } \\ \text { Serum creatinine }\end{array}\end{array}$

\section{Introduction}

Acute kidney injury (AKI) is a common complication following cardiac surgery in pediatric and adult patients alike and is strongly associated with increased short- and longterm morbidity and mortality $[1,2]$. Although extensive research has been carried out to identify risk factors for $\mathrm{AKI}$ in adults, risk factors in pediatric patients are less well known. Nonetheless, the mechanisms through which AKI can develop-and thus the risk factors-might be different in children. Furthermore, it is of great importance to identify risk factors in order to effectively implement preventive and therapeutic strategies.

In the past decades, several observational studies have been conducted to gain more insight into the pathophysiology and the risk factors of AKI in children. This paper aims to summarize the current evidence for the clinical risk factors of AKI following pediatric cardiac surgery.

\section{Methods}

\section{Eligibility criteria, databases and search strategy}

This meta-analysis was conducted in accordance with the preferred reporting items for systematic reviews metaanalyses (PRISMA) guidelines [3]. The protocol was registered in the International Prospective Register of Systematic Reviews (PROSPERO) database (registration number CRD42021262699). Studies were included if (1) the population consisted of pediatric patients ( $<18$ years old), (2) patients underwent cardiac surgery and/or surgery for congenital malformations of the heart or great vessels, (3) risk factors were compared between patients who developed AKI and those who did not, and (4) studies were prospective or retrospective observational studies or randomized controlled trials. Exclusion criteria were the following: (1) adult population, (2) non-cardiac surgery, or (3) data not available for AKI and no AKI separately.

Databases were searched for articles meeting our inclusion criteria and published by August 5, 2020: PubMed/ MEDLINE, Embase, Scopus, and reference lists of relevant articles. The detailed search terms that were used for this search are given in Supplementary Materials, Methods. The following steps were taken: (1) identification of titles of records through searching databases, (2) removal of duplicates, (3) screening and selection of abstracts, (4) assessment for eligibility through full text articles, and (5) final inclusion in the study. Studies were selected by two independent reviewers (XJ and IP). When concordance was absent, a third reviewer took the decision to include or exclude a study (JVDE).

\section{Data items}

All clinical risk factors that were reported by at least 3 studies were included in the meta-analysis. Binary risk factors included male gender, prematurity, genetic syndrome, pulmonary hypertension, cyanotic heart disease, univentricular heart, risk adjustment for congenital heart surgery 1 (RACHS-1) score $\geq 3$, prior cardiac surgery, underlying kidney condition, nephrotoxic drugs, vasopressor use, cardiopulmonary bypass (CPB) use, reoperation, and sepsis. Continuous risk factors included age (months), weight $(\mathrm{kg})$, preoperative creatinine $(\mathrm{mg} / \mathrm{dL})$, preoperative blood urea nitrogen $(\mathrm{mg} / \mathrm{dL})$, preoperative estimated glomerular filtration rate (eGFR; $\mathrm{mL} / \mathrm{min} / 1.73$ $\mathrm{m}^{2}$ ), RACHS-1 score, surgery time (min), CPB time (min), aortic cross-clamp time ( $\mathrm{min})$, and red blood cell transfusion volume $(\mathrm{mL} / \mathrm{kg})$. Nephrotoxic drugs were defined according to each individual study and could include diuretics, ACE inhibitors, antibiotics, digoxin, and various other drugs with known potential to cause kidney injury. For studies reporting interquartile ranges, the mean was estimated according to a validated formula [4]. Two independent reviewers extracted the data (BD and XJ). When concordance was absent, a third reviewer checked the data and took the final decision (JVDE). From each study, we extracted first authors' name, year of publication, country of origin, study design, years of enrollment, sample size, AKI incidence, definition of AKI, and mean age. Three main definitions for AKI currently exist; these are explained in detail in Supplementary Material, Methods.

\section{Risk of bias and certainty of evidence}

Risk of bias of the selected studies was assessed using the Cochrane risk-of-bias tool for randomized trials (RoB 2) and the risk of bias in non-randomised studies of interventions (ROBINS-I), according to study design. We also graded the level of evidence using grading of recommendation, assessment, development and evaluation (GRADE) approach for each outcome. All assessments were made independently by two reviewers (HR and $\mathrm{XJ}$ ), and any discrepancies were resolved by a third reviewer (JVDE). 


\section{Statistical analysis}

Mean differences (MD) with 95\% confidence interval (CI) and $p$ values were calculated for continuous variables. For binary variables, odds ratios (OR) with $95 \% \mathrm{CI}$ and $p$ values were considered. Chi-square test and $I^{2}$ test were performed for assessment of statistical heterogeneity [5]. In a first set of analyses, the incidences of AKI were pooled using the "metamean" function in R. Subgroup analyses were performed to check for differences in the incidence of AKI based on the definition of AKI or the use of urine output criteria.

Subsequently, the MD and OR of various risk factors were combined across the studies using two randomeffects models: an inverse-variance method ("metacont" function) and a Mantel-Haenszel method ("metabin" function), respectively [6]. The choice for random-effects models was made to account for the anticipated clinical heterogeneity of patients and surgical procedures. These models assume that the true effect sizes may differ across studies, while still allowing to assess the net effect of a certain risk factor. Funnel plots represent the analysis of publication bias, statistically analyzed by Begg and Mazumdar's rank correlation method [7] and Egger's linear regression method [8]. Subgroup analyses were conducted based on the following predefined variables: definition of AKI (AKIN, KDIGO, pRIFLE), age group (neonates, infants, toddlers, children), study design (retrospective, prospective), number of centers (single center, multicenter), and era (2001-2010, 2011-2020). Whereas a modified neonatal version of the KDIGO criteria exists, it was not investigated separately as most studies included data from patients of a broader range, thus not limited to neonates. Age groups were defined based on the mean age of all participants, as follows: neonates $<1$ month, infants 1-12 months, toddlers 12-36 months, and children $>36$ months.

Furthermore, meta-regression analyses were performed to determine whether the effects of risk factors were modulated by (1) AKI incidence (as a proxy of the overall risk profile of the study populations) or (2) age. The regression coefficient describes how the effect of the risk factor changes with a percentage increase in AKI incidence or a month increase in age, respectively. For binary risk factors, the logtransformed value of the effect of the risk factor was used in the regression model, and the exponential of the regression coefficient gives an estimate of the relative change in effect of the risk factor.

Finally, a sensitivity analysis was undertaken to identify severe AKI, which was defined as AKIN stage 3, KDIGO stage 3, or pRIFLE stage F. All analyses were completed with R Statistical Software (version 4.0.2, Foundation for Statistical Computing, Vienna, Austria).

\section{Results}

\section{Study selection and characteristics}

A total of 2,826 citations were identified, of which 106 studies were potentially relevant and retrieved as full text. Sixty-one publications fulfilled our eligibility criteria (Fig. 1). Characteristics of each study and their participants are shown in Supplementary Materials, Table S1. A total of 19,680 participants (AKI: 7257 participants; no AKI: 12,423 participants) were included from studies published from 2008 to 2020. All studies were non-randomized observational studies, except for one randomized controlled trial [9]. The pooled mean age of participants was 28.0 months (61 studies, 19,680 participants). The pRIFLE criteria [10] were implemented in 24 studies, while 17 studies used the KDIGO guidelines [11], 11 used the AKIN criteria [12], and 3 studies compared multiple definitions of AKI against one another. AKI was detected with various creatinine-based criteria in the rest of the studies. Urine output criteria were part of the definition in 17 studies, while all other studies did not take these into account. The overall internal validity of the analysis was considered moderate risk of bias (Supplementary Materials, Figures S1-S3).

\section{Synthesis of results}

\section{Incidence of AKI}

Overall, the pooled estimated incidence of AKI among children undergoing cardiac surgery was $34.3 \%$ (95\% CI $\left.30.0-38.8 \%, I^{2}=96.8 \%\right)$. Subanalyses according to definition of AKI showed similar results $(p=0.488): 37.2 \%$ (95\% CI $30.0-45.1 \%, I^{2}=94.0 \%$ ) with KDIGO, $35.6 \%$ (95\% CI $\left.29.5-42.2 \%, I^{2}=96.1 \%\right)$ with pRIFLE, and $31.4 \%$ (95\% CI $\left.22.0-42.5 \%, I^{2}=97.9 \%\right)$ with AKIN. Similarly, studies with urine output criteria reported a similar incidence of AKI $\left(37.8 \%, 95 \%\right.$ CI $\left.28.9-47.6 \%, I^{2}=96.8 \%\right)$ as those that did not $(33.1 \%, 95 \%$ CI $28.4-38.1 \%, p=0.379)$. Statistical heterogeneity could not be reduced to below $75 \%$ by conducting subanalyses based on either age group (neonates, infants, toddlers, children), study design (retrospective, prospective), number of centers (single center, multicenter), or era (2001-2010, 2011-2020).

\section{Meta-analysis of risk factors}

Results of the meta-analysis comparing risk factors between patients who developed AKI after pediatric cardiac surgery and those who did not, are summarized in Table 1. Binary risk factors that were significantly associated with AKI were 
Fig. 1 Flow diagram of studies included in data search

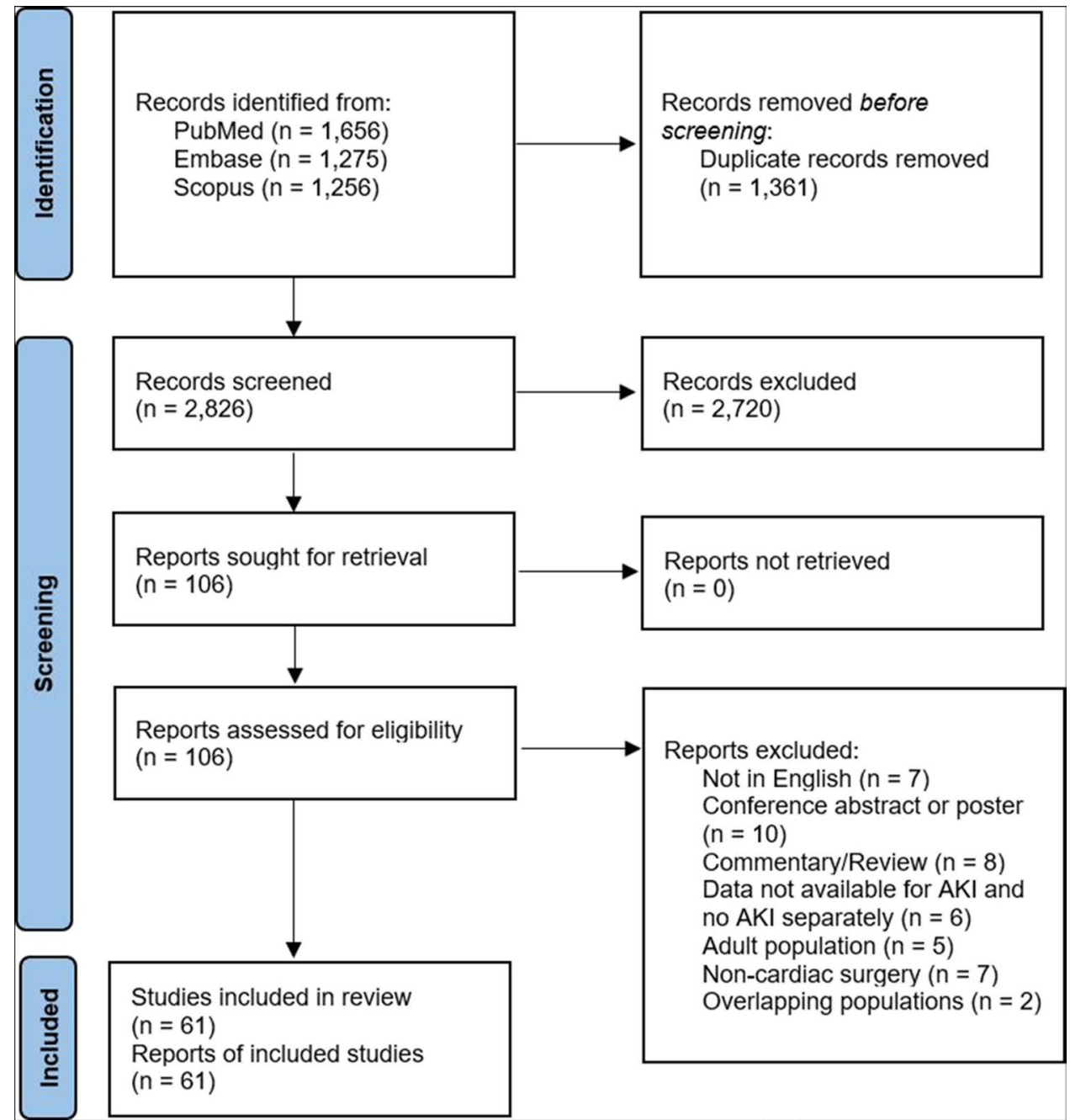

the presence of pulmonary hypertension, cyanotic heart disease, univentricular heart, RACHS-1 score $\geq 3$, nephrotoxic drugs, vasopressor use, CPB use, reoperation, and sepsis. Significant continuous risk factors included younger age, lower body weight, lower preoperative creatinine, higher preoperative eGFR, higher RACHS-1 score, longer surgery time, longer CPB time, longer aortic cross-clamp time, and higher red blood cell transfusion volume.

Funnel plot analysis (Supplementary Materials, Figures $\mathrm{S} 4-\mathrm{S} 8$ ) disclosed asymmetry around the axis for the effect of the following risk factors: nephrotoxic drugs, reoperation, age, weight, and CPB time. Consequently, publication bias related to these risk factors may be assumed. Evidence of publication bias was not found in the other risk factors.

Subanalyses revealed largely comparable results (Supplementary Materials, Table S2). The most consistent risk factor was longer CPB time, which was significantly associated with AKI in all subanalyses. Notably however, the effect of nephrotoxic drugs disappeared in almost all subanalyses. We therefore investigated if the significant effect in the overall analysis was due to a specific type of nephrotoxic drug (Supplementary Materials, Figure S9). However, no evidence could be found to establish either diuretics, ACE inhibitors, aminoglycosides, or digoxin as a risk factor for AKI following pediatric cardiac surgery.

\section{Meta-regression of the effect of risk factors}

Results of the meta-regression analyses for the effect of risk factors as a function of AKI incidence (as a proxy of overall risk profile of the study populations) and age are summarized in Supplementary Materials, Tables S3 and Table S4, respectively. We observed a significant association between AKI incidence and the effect of using CPB on the risk of developing $\mathrm{AKI}$ : for each percentage increase in AKI incidence, the effect of CPB increased by $4.1 \%$ (OR $1.041,95 \%$ CI $1.020-1.063, p=0.002)$. Furthermore, we observed a significant association between age and the effect that pulmonary hypertension exerted on the risk of 
Table 1 Meta-analysis of risk factors for AKI after pediatric cardiac surgery: summary of results

\begin{tabular}{|c|c|c|c|c|c|c|}
\hline Risk factor & $\begin{array}{l}\text { Number of } \\
\text { (sub)studies }\end{array}$ & Effect size $^{\mathrm{a}}$ & $p$ value & $I^{2}(\%)$ & $p$ value & $\begin{array}{l}\text { Certainty } \\
\text { of evidence } \\
\text { (GRADE) }\end{array}$ \\
\hline \multicolumn{7}{|l|}{ Binary risk factors } \\
\hline Male gender & 57 & $1.039(0.946 ; 1.142)$ & 0.413 & 26.4 & 0.038 & Low \\
\hline Prematurity & 8 & $1.358(0.934 ; 1.976)$ & 0.094 & 6.0 & 0.382 & Low \\
\hline Genetic syndrome & 11 & $1.131(0.949 ; 1.349)$ & 0.149 & 0.0 & 0.857 & Low \\
\hline Pulmonary hypertension & 7 & $1.772(1.279 ; 2.454)$ & 0.005 & 57.3 & 0.029 & Moderate \\
\hline Cyanotic heart disease & 18 & $3.018(2.259 ; 4.032)$ & $<0.001$ & 80.4 & $<0.001$ & High \\
\hline Univentricular heart & 14 & $1.626(1.192 ; 2.218)$ & 0.005 & 64.0 & 0.001 & High \\
\hline RACHS -1 score $\geq 3$ & 35 & $1.890(1.528 ; 2.338)$ & $<0.001$ & 77.0 & $<0.001$ & High \\
\hline Prior cardiac surgery & 15 & $1.159(0.939 ; 1.430)$ & 0.154 & 64.2 & $<0.001$ & Low \\
\hline Underlying kidney condition & 6 & $1.325(0.638 ; 2.750)$ & 0.368 & 0.0 & 0.587 & Very low \\
\hline Nephrotoxic drugs & 42 & $1.241(1.015 ; 1.517)$ & 0.036 & 61.0 & $<0.001$ & Low \\
\hline Vasopressor use & 11 & $2.824(1.995 ; 3.996)$ & $<0.001$ & 62.7 & 0.003 & High \\
\hline CPB use & 10 & $1.968(1.115 ; 3.472)$ & 0.025 & 70.5 & $<0.001$ & Moderate \\
\hline Reoperation & 9 & $5.193(2.685 ; 10.042)$ & $<0.001$ & 87.4 & $<0.001$ & Moderate \\
\hline Sepsis & 12 & $5.703(4.605 ; 7.062)$ & $<0.001$ & 6.9 & 0.378 & High \\
\hline \multicolumn{7}{|l|}{ Continuous risk factors } \\
\hline Age (months) & 63 & $-5.115(-7.414 ;-2.816)$ & $<0.001$ & 99.7 & $<0.001$ & Moderate \\
\hline Weight $(\mathrm{kg})$ & 46 & $-2.275(-3.456 ;-1.094)$ & $<0.001$ & 99.7 & $<0.001$ & Moderate \\
\hline Preoperative serum creatinine $(\mathrm{mg} / \mathrm{dL})$ & 37 & $-0.095(-0.153 ;-0.038)$ & 0.002 & 99.6 & $<0.001$ & Low \\
\hline Preoperative blood urea nitrogen (mg/dL) & 4 & $4.09(-12.797 ; 20.976)$ & 0.497 & 97.7 & $<0.001$ & Very low \\
\hline Preoperative eGFR $\left(\mathrm{mL} / \mathrm{min} / 1.73 \mathrm{~m}^{2}\right)$ & 13 & $8.019(4.157 ; 11.882)$ & 0.001 & 76.9 & $<0.001$ & High \\
\hline RACHS-1 score & 28 & $0.390(0.290 ; 0.491)$ & $<0.001$ & 76.0 & $<0.001$ & Low \\
\hline Surgery time (min) & 9 & $55.646(16.207 ; 95.084)$ & 0.012 & 99.7 & $<0.001$ & Low \\
\hline CPB time (min) & 57 & $33.471(25.852 ; 41.09)$ & $<0.001$ & 99.5 & $<0.001$ & High \\
\hline Aortic cross-clamp time (min) & 42 & $14.384(8.793 ; 19.974)$ & $<0.001$ & 99.6 & $<0.001$ & High \\
\hline Red blood cell transfusion volume $(\mathrm{mL} / \mathrm{kg})$ & 11 & $16.871(11.523 ; 22.22)$ & $<0.001$ & 99.5 & $<0.001$ & High \\
\hline
\end{tabular}

$C P B$ cardiopulmonary bypass, $e G F R$ estimated glomerular filtration rate, GRADE grading of recommendation, assessment, development and evaluation, $R A C H S-1$ risk adjustment for congenital heart surgery 1

${ }^{a}$ Effect sizes are presented as odds ratios for binary risk factors and mean differences for continuous risk factors

developing AKI: for each month increase in age, the effect of pulmonary hypertension decreased by $4.7 \%$ (OR 0.953 , 95\% CI 0.931-0.975, $p=0.003)$. Similarly, a significant association was found between AKI incidence and the effect of prior cardiac surgery on the risk of developing AKI: for each percentage increase in AKI incidence, the effect of prior cardiac surgery decreased by $2.9 \%$ (OR $0.971,95 \% \mathrm{CI}$ $0.949-0.994, p=0.018)$. Lastly, increasing age was found to increase the effect sizes of age (MD $-0.374,95 \% \mathrm{CI}$ $-0.520,-0.229, p<0.001)$ and weight (MD $-0.058,95 \%$ CI $-0.111,-0.005, p=0.034)$. No significant associations were found for other risk factors.

\section{Risk factors of severe AKI}

The sensitivity analysis including risk factors of severe AKI was only possible for male gender and nephrotoxic drugs, and was unable to find a significant association of either of these with severe AKI (Supplementary Materials, Table S5). All continuous variables, except for preoperative blood urea nitrogen and surgery time, could be evaluated and showed comparable results. However, RACHS-1 score was not significantly associated with severe AKI and lower-rather than higher-preoperative eGFR was found in patients with severe AKI.

\section{Discussion}

\section{Summary of evidence}

Although risk factors for cardiac surgery-associated AKI in adults are well known, in children these had not been subjected to a systematic investigation before. The present meta-analysis, including 19,680 participants from 61 studies, identified risk factors that were significantly associated with 
AKI in pediatric patients following cardiac surgery. Binary risk factors included the presence of pulmonary hypertension, cyanotic heart disease, univentricular heart, RACHS-1 score $\geq 3$, nephrotoxic drugs, vasopressor use, CPB use, reoperation, and sepsis (Fig. 2). Continuous risk factors were younger age, lower body weight, lower preoperative creatinine, higher preoperative eGFR, higher RACHS-1 score, longer surgery time, longer CPB time, longer aortic cross-clamp time, and higher red blood cell transfusion volume. Sensitivity analyses revealed largely comparable results, except for the fact that the effect of nephrotoxic drugs disappeared in all subanalyses and nephrotoxic drugs and RACHS-1 score disappeared in the analysis of severe AKI. Furthermore, lower-rather than higher-preoperative eGFR was associated with severe AKI. In summary, our extensive analysis identified risk factors that could serve for risk stratification and timely implementation of effective preventive and therapeutic strategies for AKI.

\section{Kidney risk factors}

The underlying pathophysiology of cardiac surgery-associated AKI in children differs from that in adults. Several features of pediatric kidney physiology are expected to play a role. First of all, kidney maturation is not completed until the age of 2-3 years, resulting in a high susceptibility to inflammatory and ischemic changes [13]. Indeed, having immature kidneys with little to no possibilities to compensate for kidney ischemia, neonates and infants in particular are at elevated risk of developing AKI. Secondly, considerable changes in the kidney vasculature occur in young children. At birth, only $2.5-4.0 \%$ of the cardiac output passes through the kidneys, which is approximately 5 to tenfold lower than that in adults [13]. As systemic arteriolar resistance increases and kidney vascular resistance decreases early in life, a rapid increase in kidney perfusion pressure and thus kidney blood flow occurs [13]. These changes are likely to cause kidney stress and thus might constitute one of the mechanisms of AKI that are unique to children.

Interestingly, our main meta-analysis found that low preoperative creatinine and high preoperative eGFR were associated with AKI. This is in contrast to studies in adults, where low-rather than high-preoperative eGFR was found to be a risk factor for AKI [14]. The above described physiological changes might help explain this apparent paradox: the rapid increase in kidney blood flow also increases eGFR [15]. As such, relative hyperfiltration in these children might be a risk factor of AKI because it indicates kidney stress. Although the exact mechanisms could not be determined in the present meta-analysis, this hypothesis merits further investigation. In the sensitivity analyses, low preoperative eGFR was associated with severe AKI, which is again in
Fig. 2 Forest plots summarizing main findings. $C I$ confidence interval, $e G F R$ estimated glomerular filtration rate, $R A C H S-1$ risk adjustment for congenital heart surgery 1

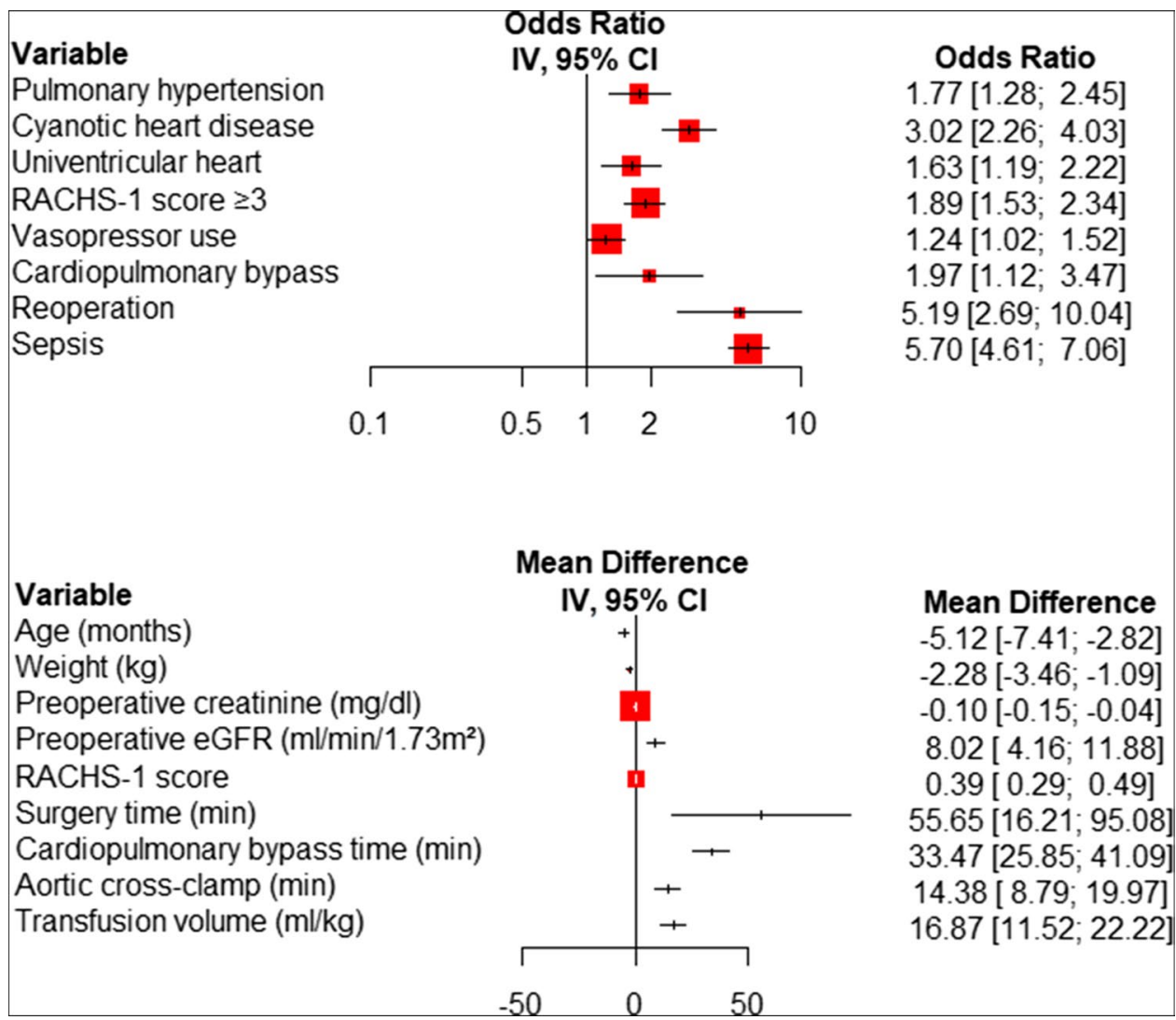


agreement with adult studies. However, low preoperative creatinine was also found in patients with severe AKI. Another explanation has therefore been raised by Algaze et al. [16]. They suggested that this finding might result from the definition of AKI itself, as current criteria mainly focus on relative increases in serum creatinine ( $\mathrm{SCr}$ ). In patients with lower preoperative $\mathrm{SCr}$ levels, a smaller increase in absolute terms is required to achieve a 1.5 -fold increase. As a result, these patients are more likely to be detected as having AKI by current criteria. Conversely, children with higher preoperative $\mathrm{SCr}$ levels are at risk of developing AKI that remains undetected or is underestimated, but might nonetheless have systemic effects similar to more severe injury stages [17]. Both situations are concerning because $\mathrm{SCr}$ levels are highly variable in children: these are highest in neonates, then decrease rapidly in the first two months of life, and subsequently increase again before reaching kidney maturation at the age of $2-3$ years old $[18,19]$.

Currently, three main criteria are used to define AKI: AKIN, KDIGO, and pRIFLE. Since the heterogeneous use of these criteria in the included studies is a fundamental issue which complicates any comparison between studies, we conducted subanalyses according to AKI definition in order to reach higher comparability and generalizability. We found that the different definitions of AKI did not influence the observed incidence of AKI. On the other hand, different results were observed for some risk factors depending on the definition of AKI. Although some of the interaction terms in the Supplementary Material, Table S2 might be the result of small sample sizes in the subanalyses, the differences remain apparent in some of the risk factors for which at least 8 studies were available in each of the subgroups. For example, preoperative creatinine was associated with AKI in studies using KDIGO and pRIFLE criteria, but not in those using the AKIN criteria. Similarly, age was not a significant risk factor in a subanalysis of studies using the AKIN criteria, even though the mean age of the patients in these studies was comparable to that in studies using KDIGO and pRIFLE criteria. These results emphasize the need for more standardized AKI definitions based on more sensitive and specific biomarkers than creatinine and adapted to the unique kidney physiology in children.

On a further note, most studies have omitted the urine output contribution in the definition of AKI. Adult studies have reported that the current criterion of $0.5 \mathrm{ml} / \mathrm{kg} / \mathrm{hour}$ for $6 \mathrm{~h}$ is overly sensitive and not prognostically aligned with the $\mathrm{SCr}$ criteria [20]. The AWARE study [21] in critically ill children and young adolescents and the AWAKEN study [22] in neonates reported that the diagnosis of AKI would have been missed if $\mathrm{SCr}$ criteria had been used alone in $10.7 \%$ (355/3318) and 6.6\% (133/2022) of patients, respectively. While the present meta-analysis could not confirm this, these prior findings suggest that use of the urine output criteria might influence the incidence of AKI. Fine-tuning of the urine output criteria, such as lowering the threshold [20], within different populations might be needed to ensure that the definition of AKI remains reflective of outcomes.

Although nephrotoxic drugs were identified as risk factors in the overall analysis, their effect disappeared in almost all subanalyses. Even in a subgroup analysis based on drug type (diuretics, ACE inhibitors, aminoglycosides, or digoxin), no significant contribution to the risk of developing AKI could be observed. This could be because children have fewer comorbidities, less severe pre-existing kidney dysfunction, and higher kidney functional reserve, and are thus less susceptible to the kidney side effects of these drugs [23]. That scenario, although it can only be regarded as a hypothesis, would imply that the avoidance of these nephrotoxic drugs has little efficacy as a preventive strategy. Nonetheless, we consider it essential to introduce a word of caution here. Indeed, various other studies in children have demonstrated nephrotoxic effects and an increased risk of AKI with the use of certain drugs, such as for instance the combination of vancomycin with piperacillin/tazobactam [24]. Furthermore, most clinical practice guidelines recommend to discontinue, lower or monitor the doses of nephrotoxic drugs prior to cardiac surgery [11]. Therefore, the negative results of this study regarding the risks of nephrotoxic drugs in pediatric patients undergoing cardiac surgery certainly do not imply that these drugs can be safely continued. The absence of evidence is not the same as evidence of absence.

\section{Systemic risk factors}

The most consistent risk factor in our analysis was longer CPB time, which remained significantly associated with AKI in all subanalyses. From a pathophysiological perspective, CPB can cause activation of coagulation pathways, ischemia-reperfusion injury, systemic inflammation, and reduction of GFR [25]. The adverse effects of CPB are particularly pronounced in young pediatric patients due to their hematologic immaturity and high levels of thrombin generation during and after CPB [26]. Additionally, the aforementioned factors such as immature and small kidneys result in an increased susceptibility to reperfusion injury following CPB [13]. Interestingly, the effect of using CPB was found to increase with higher $\mathrm{AKI}$ incidence in our meta-regression analyses, suggesting that CPB plays an especially important role in populations that are already at high risk of developing AKI. Although not included in the meta-analysis, selective cerebral perfusion [27] and deep hypothermic circulatory arrest [28] were both associated with increased risk of AKI, independently from CPB and other risk factors.

Sepsis was the strongest risk factor in our meta-analysis $(\mathrm{OR}=5.70)$. $\mathrm{CPB}$ might be one contributing factor, with contact of blood components with the artificial surface of the 
bypass circuit, ischemia-reperfusion injury, endotoxemia, operative trauma, non-pulsatile blood flow, and pre-existing left ventricular dysfunction all being causes of systemic inflammatory response syndrome (SIRS) [29]. The resulting spectrum of injury to multiple organs can lead to an increased susceptibility to infections and subsequent sepsis [30]. In addition, many of these children stay long times in hospital or intensive care unit settings, which itself is a major risk factor for sepsis [31]. Sepsis on its own is a major cause of AKI in children and is characterized by high mortality [32]. The pathophysiology likely involves an interplay of cytokine-mediated capillary leakage decreased intravascular volumes, edema, and disturbances in the microcirculation [33].

Age was also found to be a significant risk factor, and risk factors had different effects depending on age. Similar results have been reported by $\mathrm{Li}$ et al. [34]. In a meta-analysis of 9925 patients with congenital heart disease (CHD) from 30 studies, they found that lower age was significantly associated with AKI incidence rate. The effect of age on the development of AKI might be related to immaturity of the kidneys in younger children and a predisposition to ischemia and coagulation as described above. In addition, our metaanalysis found that decreasing age was found to decrease the effect size of age and weight. However, we assume that these findings are primarily a statistical phenomenon: the potential variability of age and weight between individuals within a study population increases with age, such that the mean difference between cases and controls tends to increase.

\section{Risk factors related to congenital heart disease}

Apart from the kidney and systemic mechanisms described above, CHD itself increases the risk of developing AKI following pediatric cardiac surgery. This is illustrated by the fact that cyanotic CHD and univentricular heart were both significantly associated with AKI. Nephropathy has been reported as a common feature in cyanotic CHD and its dominant feature is glomerular damage, which is related to the duration of cyanosis [35]. Furthermore, chronic kidney disease or dysfunction is evident in half of the patients after the Fontan operation once they reach adolescence and early adulthood [36]. As a result of ongoing damage to the kidneys due to CHD itself, these patients might thus not only be at risk of experiencing AKI but also of developing chronic kidney disease later in life. Therefore, kidney function of patients with CHD, and especially those with cyanosis or a univentricular heart, warrants close follow-up.

Pulmonary hypertension is a common complication of CHD that is generally caused by pressure overload and conditions with intracardiac shunting such as ventricular septal defects, but can also result following correction of CHD [37]. In addition, pulmonary hypertension in this population might occur in the context of primary pulmonary vascular changes, effects of prematurity, or other anatomic abnormalities. As shown in our meta-analysis, pulmonary hypertension was associated with AKI following cardiac surgery. There is some consensus in the literature that the primary mechanism through which pulmonary hypertension leads to $\mathrm{AKI}$ is through venous congestion and an increase in central venous pressure [38]. This in turn decreases effective kidney perfusion, triggering activation of the renin-angiotensin-aldosterone system, the sympathetic nervous system, and pro-inflammatory cascades [39]. Our meta-regression analysis also revealed that this effect was mainly seen in younger patients. A possible explanation could be provided by the fact that pulmonary hypertension in younger children is associated with more severe types of simple shunt lesions (large atrial or ventricular septal defects) or worse comorbidities such as concomitant chronic lung disease [40]. Another explanation might be related to the typical low compliance of the right ventricle in neonates and infants. A right ventricle with low compliance will be more prone to conduct pressure retrogradely; it could thereby lead to venous congestion of the kidney, which is a known mechanism of AKI [41].

\section{Perspectives for future research and clinical practice}

The exact pathophysiology of postoperative AKI in pediatric patients undergoing cardiac surgery is not fully understood yet and is most likely multifactorial. However, our metaanalysis has identified important clinical risk factors for AKI development. Early identification of risk factors for AKI provides opportunities to implement preventive strategies and to start close monitoring. Furthermore, a patient's risk profile for AKI might inform choices of surgical techniques. In light of our findings, future research should focus on the modification of risk factors such as CPB time and vasopressor use. For example, the development of off-pump cardiac surgery in recent years holds promise as an alternative to CPB for some types of CHD.

As discussed above, current definitions of AKI have considerable limitations. In addition, $\mathrm{SCr}$, being in fact a marker of kidney function rather than injury, only increases once $50 \%$ of all nephrons have been destroyed; it therefore considerably lags behind [11]. The recent development of more sensitive biomarkers other than creatinine holds promise to better and more timely identify patients with prognostically relevant kidney injury $[42,43]$.

Nonetheless, identification is only one aspect, while AKI after pediatric cardiac surgery has shown to be frustratingly difficult to prevent and treat. No firm evidence currently exists about the protective role of various pharmacological interventions [44]. Furthermore, evidence of progressive kidney injury in these children several years after the AKI episode has been reported, which cannot be readily 
reversed [45]. As a result, the prevalence of chronic kidney disease among adults with CHD remains high at 30-50\% [46]. Therefore, increased research efforts should be invested in the discovery of novel strategies to effectively prevent and treat AKI. The knowledge obtained from these studies may contribute to creating successful pre-, peri- and postoperative protocols.

\section{Limitation and sources of heterogeneity}

There are certain limitations that need to be considered when interpreting the results. Firstly, this meta-analysis included mostly observational studies. Consequently, our findings are limited to significant associations and cannot demonstrate causality. Secondly, sample sizes in some subgroups were relatively small, which limits the interpretation of those subanalyses. In the third place, our funnel plot analysis revealed asymmetry around the axis for several risk factors, including nephrotoxic drugs, reoperation age, weight and CPB time. As a result, the possibility of publication bias related to these outcomes should be taken into consideration. Finally, statistical and clinical heterogeneity complicate the interpretation of the results. As addressed before, differences in definitions of AKI may lead to statistical heterogeneity. It has also been demonstrated how certain risk factors might be less or more important depending on age and overall risk profile (as reflected by AKI incidence in the meta-regression analyses). Other sources of heterogeneity might include study design, numbers of centers, era, and underlying type of CHD. For example, our subanalyses revealed that the effect of RACHS-1 score (both the binary and continuous variable) was larger in single center studies, potentially because variations in surgical experience become diluted in multicenter studies. Furthermore, the difference in surgery time between children with and without AKI was larger in studies published in 2001-2011 than in 2011-2020, suggesting continuous improvements in surgical technique. Lastly, differences depending on study design might be related to the limitations of retrospective enrollment of patients and collection of data.

\section{Conclusions}

Based on current evidence from observational studies, this meta-analysis identified clinical risk factors associated with $\mathrm{AKI}$ in pediatric patients undergoing cardiac surgery. These constitute risk factors that could serve for risk stratification, prevention, and timely intervention for AKI in children. Future research should aim to establish protocols for kidney follow-up of patients with CHD, develop strategies to effectively prevent $\mathrm{AKI}$, and investigate biomarkers for improved diagnosis of AKI.
Supplementary Information The online version contains supplementary material available at https://doi.org/10.1007/s00467-021-05297-0.

Acknowledgements J. Van den Eynde was supported by the Belgian American Educational Foundation.

Author contributions JVDE: Conceptualization; Data curation; Formal analysis; Investigation; Methodology; Project administration; Software; Validation; Visualization; Writing_original draft; Writing-review \& editing. BD: Conceptualization; Data curation; Investigation; Writing —original draft; Writing — review \& editing. XJ: Conceptualization; Data curation; Investigation; Writing — original draft; Writing-review \& editing. IP: Conceptualization; Data curation; Investigation; Writing —original draft; Writing — review \& editing. HR: Conceptualization; Data curation; Investigation; Writing — original draft; Writingreview \& editing. MG: Conceptualization; Investigation; Supervision; Writing-review \& editing. SK: Conceptualization; Investigation; Supervision; Writing-review \& editing. DM: Conceptualization; Investigation; Supervision; Writing—review \& editing.

Funding This research received no specific grant from any funding agency in the public, commercial, or not-for-profit sectors.

Data availability The datasets generated during and/or analysed during the current study are available from the corresponding author on reasonable request.

Code availability The code used for this study will be made available from the corresponding author, upon reasonable request.

\section{Declarations}

Conflict of interest M. Gewillig is proctor for Edwards and Medtronic. S. Kutty is consultant for GE Healthcare. All other authors declare that they have no competing interests.

\section{References}

1. Hoste EAJ, Kellum JA, Selby NM, Zarbock A, Palevsky PM, Bagshaw SM, Goldstein SL, Cerdá J, Chawla LS (2018) Global epidemiology and outcomes of acute kidney injury. Nat Rev Nephrol 14:607-625

2. Van den Eynde J, Rotbi H, Gewillig M, Kutty S, Allegaert K (2021) In-Hospital outcomes of acute kidney injury after pediatric cardiac surgery : a meta-analysis. Front Pediatr 9:733744. https:// doi.org/10.3389/fped.2021.733744

3. Page MJ, McKenzie JE, Bossuyt PM, Boutron I et al (2021) The PRISMA 2020 statement: an updated guideline for reporting systematic reviews. BMJ 372:n71

4. Hozo SP, Djulbegovic B, Hozo I (2005) Estimating the mean and variance from the median, range, and the size of a sample. BMC Med Res Methodol 5:13. https://doi.org/10.1186/1471-2288-5-13

5. Higgins JPT, Thompson SG, Deeks JJ, Altman DG (2003) Measuring inconsistency in meta-analyses. Br Med J 327:557-560

6. DerSimonian R, Kacker R (2007) Random-effects model for meta-analysis of clinical trials: an update. Contemp Clin Trials 28:105-114. https://doi.org/10.1016/j.cct.2006.04.004

7. Begg CB, Mazumdar M (1994) Operating characteristics of a rank correlation test for publication bias. Biometrics 50:1088. https:// doi.org/10.2307/2533446 
8. Egger M, Smith GD, Schneider M, Minder C (1997) Bias in metaanalysis detected by a simple, graphical test. Br Med J 315:629634. https://doi.org/10.1136/bmj.316.7129.469

9. Blinder JJ, Asaro LA, Wypij D, Selewski DT, Agus MSD, Gaies M, Ferguson MA (2017) Acute kidney injury after pediatric cardiac surgery: a secondary analysis of the safe pediatric euglycemia after cardiac surgery trial. Pediatr Crit Care Med 18:638-646. https://doi.org/10.1097/PCC.0000000000001185

10. Akcan-Arikan A, Zappitelli M, Loftis LL, Washburn KK, Jefferson LS, Goldstein SL (2007) Modified RIFLE criteria in critically ill children with acute kidney injury. Kidney Int 71:1028-1035. https://doi.org/10.1038/sj.ki.5002231

11. Khwaja A (2012) KDIGO clinical practice guidelines for acute kidney injury. Nephron 120:c179-c184. https://doi.org/10.1159/ 000339789

12. Mehta RL, Kellum JA, Shah SV, Molitoris BA et al (2007) Acute kidney injury network: report of an initiative to improve outcomes in acute kidney injury. Crit Care 11:R31. https://doi.org/10.1186/ cc5713

13. Selewski DT, Charlton JR, Jetton JG, Guillet R, Mhanna MJ, Askenazi DJ, Kent AL (2015) Neonatal acute kidney injury. Pediatrics 136:e463-e473. https://doi.org/10.1542/peds.2014-3819

14. Mooney JF, Ranasinghe I, Chow CK, Perkovic V, Barzi F, Zoungas S, Holzmann MJ, Welten GM, Biancari F, Wu VC, Tan TC, Cass A, Hillis GS (2013) Preoperative estimates of glomerular filtration rate as predictors of outcome after surgery: a systematic review and meta-analysis. Anesthesiology 118:809-824

15. Brenner BM, Lawler EV, Mackenzie HS (1996) The hyperfiltration theory: a paradigm shift in nephrology. Kidney Int 49:1774-1777

16. Algaze CA, Koth AM, Faberowski LW, Hanley FL, Krawczeski CD, Axelrod DM (2017) Acute kidney injury in patients undergoing the extracardiac Fontan operation with and without the use of cardiopulmonary bypass. Pediatr Crit Care Med 18:34-43. https:// doi.org/10.1097/PCC.0000000000000984

17. Bojan M, Lopez-Lopez V, Pouard P, Falissard B, Journois D (2013) Limitations of early serum creatinine variations for the assessment of kidney injury in neonates and infants with cardiac surgery. PLoS One 8:e79308. https://doi.org/10.1371/JOURNAL. PONE.0079308

18. Springate JE, Christensen SL, Feld LG (1992) Serum creatinine level and renal function in children. Am J Dis Child 146:12321235. https://doi.org/10.1001/archpedi.1992.02160220118036

19. Boer DP, De Rijke YB, Hop WC, Cransberg K, Dorresteijn EM (2010) Reference values for serum creatinine in children younger than 1 year of age. Pediatr Nephrol 25:2107-2113. https://doi.org/ 10.1007/s00467-010-1533-y

20. MdRalib A, Pickering JW, Shaw GM, Endre ZH (2013) The urine output definition of acute kidney injury is too liberal. Crit Care 173:1-11. https://doi.org/10.1186/CC12784

21. Kaddourah A, Basu RK, Bagshaw SM, Goldstein SL (2016) Epidemiology of acute kidney injury in critically ill children and young adults. N Engl J Med 376:11-20. https://doi.org/10.1056/ NEJMOA1611391

22. Jetton JG, Boohaker LJ, Sethi SK, Wazir S et al (2017) Incidence and outcomes of neonatal acute kidney injury (AWAKEN): a multicentre, multinational, observational cohort study. Lancet Child Adolesc Heal 1:184-194. https://doi.org/10.1016/S2352-4642(17) 30069-X

23. Fuhrman DY (2021) The role of renal functional reserve in predicting acute kidney injury. Crit Care Clin 37:399-407. https:// doi.org/10.1016/J.CCC.2020.11.008

24. Kalligeros M, Karageorgos SA, Shehadeh F, Zacharioudakis IM, Mylonakis E (2019) The association of acute kidney injury with the concomitant use of vancomycin and piperacillin-tazobactam in children: a systematic review and meta-analysis. Antimicrob Agents Chemother 63:e01572-01519. https://doi.org/10.1128/ AAC.01572-19

25. Paparella D, Yau TM, Young E (2002) Cardiopulmonary bypass induced inflammation: pathophysiology and treatment. An update. Eur J Cardiothorac Surg 21:232-244

26. Eaton MP, Iannoli EM (2011) Coagulation considerations for infants and children undergoing cardiopulmonary bypass. Pediatr Anesth 21:31-42. https://doi.org/10.1111/j.1460-9592.2010. 03467.x

27. Piggott KD, Soni M, Decampli WM, Ramirez JA, Holbein D, Fakioglu H, Blanco CJ, Pourmoghadam KK (2015) Acute kidney injury and fluid overload in neonates following surgery for congenital heart disease. World J Pediatr Congenit Heart Surg 6:401-406. https://doi.org/10.1177/2150135115586814

28. Morgan CJ, Zappitelli M, Robertson CMT, Alton GY, Sauve RS, Joffe AR, Ross DB, Rebeyka IM (2013) Risk factors for and outcomes of acute kidney injury in neonates undergoing complex cardiac surgery. J Pediatr 162:120-127.e1. https://doi.org/10.1016/j. jpeds.2012.06.054

29. Boehne M, Sasse M, Karch A, Dziuba F, Horke A, Kaussen T, Mikolajczyk R, Beerbaum P, Jack T (2017) Systemic inflammatory response syndrome after pediatric congenital heart surgery: Incidence, risk factors, and clinical outcome. J Card Surg 32:116125. https://doi.org/10.1111/jocs. 12879

30. Rosner MH, Okusa MD (2006) Acute kidney injury associated with cardiac surgery. Clin J Am Soc Nephrol 1:19-32. https://doi. org/10.2215/CJN.00240605

31. Wheeler DS, Jeffries HE, Zimmerman JJ, Wong HR, Carcillo JA (2011) Sepsis in the pediatric cardiac intensive care unit. World J Pediatr Congenit Heart Surg 2:393. https://doi.org/10.1177/21501 35111403781

32. Fitzgerald JC, Basu RK, Akcan-Arikan A, Izquierdo LM et al (2016) Acute kidney injury in pediatric severe sepsis: an independent risk factor for death and new disability. Crit Care Med 44:2241-2250. https://doi.org/10.1097/CCM.0000000000002007

33. Zarjou A, Agarwal A (2011) Sepsis and acute kidney injury. J Am Soc Nephrol 22:999-1006. https://doi.org/10.1681/ASN.20100 50484

34. Li D, Niu Z, Huang Q, Sheng W, Wang T (2020) A meta-analysis of the incidence rate of postoperative acute kidney injury in patients with congenital heart disease. BMC Nephrol 21:350. https://doi.org/10.1186/s12882-020-02005-2

35. Dittrich S, Haas N, Bührer C, Müller C, Dähnert I, Lange P (2007) Renal impairment in patients with long-standing cyanotic congenital heart disease. Acta Paediatr 87:949-954. https://doi.org/ 10.1111/j.1651-2227.1998.tb01764.x

36. Lee D, Levin A, Kiess M, Sexsmith G, Chakrabarti S, Barlow A, Human D, Grewal J (2018) Chronic kidney damage in the adult Fontan population. Int J Cardiol 257:62-66. https://doi.org/10. 1016/j.ijcard.2017.11.118

37. Abman SH, Hansmann G, Archer SL, Ivy DD et al (2015) Pediatric pulmonary hypertension. Circulation 132:2037-2099

38. Ronco C, McCullough P, Anker SD, Anand I et al (2010) Cardiorenal syndromes: report from the consensus conference of the acute dialysis quality initiative. Eur Heart J 31:703-711. https:// doi.org/10.1093/EURHEARTJ/EHP507

39. Naranjo M, Lo KB, Mezue K, Rangaswami J (2019) Effects of pulmonary hypertension and right ventricular function in short and long-term kidney function. Curr Cardiol Rev 15:3. https:// doi.org/10.2174/1573403X14666181008154215

40 Kozlik-Feldmann R, Hansmann G, Bonnet D, Schranz D, Apitz C, Michel-Behnke I (2016) Pulmonary hypertension in children with congenital heart disease (PAH-CHD, PPHVD-CHD). Expert consensus statement on the diagnosis and treatment of paediatric 
pulmonary hypertension. The European paediatric pulmonary vascular disease network, endorsed by ISHLT and DGPK. Heart 102:42-48. https://doi.org/10.1136/heartjnl-2015-308378

41. Ostermann M, Liu K (2017) Pathophysiology of AKI. Best Pract Res Clin Anaesthesiol 31:305-314

42. Mårtensson J, Martling CR, Bell M (2012) Novel biomarkers of acute kidney injury and failure: clinical applicability. $\mathrm{Br} \mathrm{J}$ Anaesth 109:843-850

43. Coca SG, Yalavarthy R, Concato J, Parikh CR (2008) Biomarkers for the diagnosis and risk stratification of acute kidney injury: a systematic review. Kidney Int 73:1008-1016

44. Bellos I, Iliopoulos DC, Perrea DN (2019) Pharmacological interventions for the prevention of acute kidney injury after pediatric cardiac surgery: a network meta-analysis. Clin Exp Nephrol 23:782-791. https://doi.org/10.1007/s10157-019-01706-9

45. Cooper DS, Claes D, Goldstein SL, Bennett MR, Ma Q, Devarajan P, Krawczeski CD (2016) Follow-up renal assessment of injury long-term after acute kidney injury (FRAIL-AKI). Clin J Am Soc Nephrol 11:21-29. https://doi.org/10.2215/CJN.04240415

46. Morgan C, Al-Aklabi M, Garcia Guerra G (2015) Chronic kidney disease in congenital heart disease patients: a narrative review of evidence. Can J Kidney Heal Dis 2:27. https://doi.org/10.1186/ s40697-015-0063-8

Publisher's note Springer Nature remains neutral with regard to jurisdictional claims in published maps and institutional affiliations.

\section{Authors and Affiliations}

\section{Jef Van den Eynde ${ }^{1,2}$ D $\cdot$ Boris Delpire ${ }^{2} \cdot$ Xander Jacquemyn $^{2} \cdot$ Ismat Pardi $^{2} \cdot$ Hajar Rotbi $^{3,4} \cdot$ Marc Gewillig $^{2,5}$. Shelby Kutty ${ }^{1} \cdot$ Djalila Mekahli $^{6,7}$}

1 Helen B. Taussig Heart Center, The Johns Hopkins Hospital and School of Medicine, Baltimore, USA

2 Department of Cardiovascular Sciences, University Hospitals Leuven, KU Leuven, Herestraat 49, 3000 Leuven, Belgium

3 Faculty of Medicine, Radboud University, Nijmegen, The Netherlands

4 Department of Physiology, Radboud University Medical Center, Radboud Institute for Health Sciences, Nijmegen, The Netherlands
5 Pediatric Cardiology, University Hospitals Leuven, Leuven, Belgium

6 Department of Pediatric Nephrology, University Hospitals of Leuven, Leuven, Belgium

7 PKD Research Group, GPURE, Department of Development and Regeneration, KU Leuven, Leuven, Belgium 